\title{
Correction to: Incidence of Hepatocellular Carcinoma in Primary Biliary Cholangitis: A Systematic Review and Meta-Analysis
}

\author{
Yamini Natarajan ${ }^{1}$ (1) $\cdot$ Aylin Tansel ${ }^{1} \cdot$ Parth Patel $^{1} \cdot$ Ikenna K. Emelogu $^{1} \cdot$ Richa Shukla $^{1} \cdot$ Zeeshan Qureshi $^{1}$. \\ Hashem B. El-Serag ${ }^{1,2,3,4} \cdot$ Aaron P. Thrift ${ }^{1,4} \cdot$ Fasiha Kanwal $^{1,2,3,4}$
}

Published online: 29 September 2020

(c) This is a U.S. Government work and not under copyright protection in the US; foreign copyright protection may apply 2020

\section{Correction to: Digestive Diseases and Sciences https://doi.org/10.1007/s10620-020-06498-7}

The original version of the article unfortunately contained an error in the first name and the surname of the fourth author, Dr. Ikenna K. Emelogu in the author group. The author name was incorrectly published as 'Kingsley Emologu' and the corrected name is 'Ikenna K. Emelogu'.

Publisher's Note Springer Nature remains neutral with regard to jurisdictional claims in published maps and institutional affiliations.

The original article can be found online at https://doi.org/10.1007/ s10620-020-06498-7.

Yamini Natarajan ynataraj@bcm.edu

1 Section of Gastroenterology and Hepatology and Clinical Epidemiology and Comparative Effectiveness Program in the Health Services Research, Michael E. DeBakey VA Medical Center, Baylor College of Medicine, 2002 Holcombe Blvd (MS152), Houston, TX 77030, USA

2 Clinical Epidemiology and Comparative Effectiveness Program, Section of Health Services Research (IQuESt), Michael E. DeBakey VA Medical Center, Baylor College of Medicine, Houston, TX, USA

3 Texas Medical Center Digestive Disease Center, Houston, TX, USA

4 Dan L Duncan Comprehensive Cancer Center at Baylor College of Medicine, Houston, TX, USA 